\title{
Adjustable and distributionally robust chance-constrained economic dispatch considering wind power uncertainty
}

\author{
Xin FANG ${ }^{1}$, Bri-Mathias HODGE ${ }^{1}$, Fangxing $\mathbf{L I}^{2}$ (D), \\ Ershun DU ${ }^{3}$, Chongqing KANG ${ }^{3}$
}

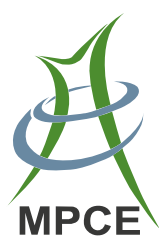

\begin{abstract}
This paper proposes an adjustable and distributionally robust chance-constrained (ADRCC) optimal power flow (OPF) model for economic dispatch considering wind power forecasting uncertainty. The proposed ADRCC-OPF model is distributionally robust because the uncertainties of the wind power forecasting are represented only by their first- and second-order moments instead of a specific distribution assumption. The proposed model is adjustable because it is formulated as a second-order cone programming (SOCP) model with an adjustable coefficient. This coefficient can control the robustness of the chance constraints, which may be set up for the Gaussian distribution, symmetrically distributional robustness, or
\end{abstract}

CrossCheck date: 25 January 2019

Received: 2 June 2018/Accepted: 25 January 2019/Published online: 24 April 2019

(C) The Author(s) 2019

$\triangle$ Fangxing LI

fli6@utk.edu

Xin FANG

Xin.Fang@nrel.gov

Bri-Mathias HODGE

Bri.Mathias.Hodge@nrel.gov

Ershun DU

des13@mails.tsinghua.edu.cn

Chongqing KANG

cqkang@tsinghua.edu.cn

1 National Renewable Energy Laboratory, Golden, CO 80401, USA

2 Department of Electrical Engineering and Computer Science, The University of Tennessee, Knoxville, TN 37996, USA

3 State Key Laboratory of Power Systems, Department of Electrical Engineering, Tsinghua University, Beijing 100084, China distributionally robust cases considering wind forecasting uncertainty. The conservativeness of the ADRCC-OPF model is analyzed and compared with the actual distribution data of wind forecasting error. The system operators can choose an appropriate adjustable coefficient to tradeoff between the economics and system security.

Keywords Economic dispatch, Adjustable and distributionally robust chance-constrained optimization, Wind power forecasting, Uncertainty

\section{Introduction}

Recently, the increased deployment of wind power has brought challenges to electric power systems operation because of its power output uncertainty. To reliably operate the system, stochastic optimization, such as chance-constrained optimal power flow (CC-OPF), has been introduced $[1,2]$ to minimize the cost of system generation while maintaining reliability and avoiding constraint violations within a defined risk level. Previously, the Gaussian distribution was used to represent wind power forecasting uncertainty [3,4], but this assumption might lead to an estimation error for the system condition. Distributionally robust CC-OPF (DRCC-OPF) is proposed in [3], which needed only the forecasting mean and variance information, and can lead to a conservative dispatch and high generation cost because the result is robust for any forecasting errors distribution. This violation probability is either higher or lower than the predefined violation level using the predefined distribution assumptions. A higher violation means the system risk level is higher than the preferred level. A lower violation means that the system cost is higher than the optimal value. Therefore, choosing 
an appropriate coefficient can give a trade-off between the system reliability and cost. In this letter, an adjustable and distributionally robust chance-constrained (ADRCC) optimal power flow (OPF) model is proposed to obtain this optimal coefficient.

When the wind power forecasting uncertainty is taken into consideration, the CC-OPF model is given by:

$$
\begin{array}{ll}
\min & \sum_{i=1}^{N} c_{i} G_{\text {exp }, i} \\
\text { s.t. } & \sum_{i=1}^{N}\left(G_{i}+P_{i}\right)-\sum_{i=1}^{N} D_{i}=0 \\
& \operatorname{Pr}\left(\sum_{i=1}^{N} G S F_{l, i} \cdot\left(G_{i}+P_{i}-D_{i}\right) \leq L U_{l}\right) \geq 1-\epsilon \\
& \operatorname{Pr}\left(-L U_{l} \leq \sum_{i=1}^{N} G S F_{l, i} \cdot\left(G_{i}+P_{i}-D_{i}\right)\right) \geq 1-\epsilon \\
& \operatorname{Pr}\left(G_{i} \leq G_{i, \max }\right) \geq 1-\epsilon \\
& \operatorname{Pr}\left(G_{i, \min } \leq G_{i}\right) \geq 1-\epsilon \\
& G_{i}=G_{\text {exp }, i}+\Delta G_{i} \\
& P_{i}=P_{\text {exp }, i}+\Delta P_{i} \\
& \Delta G_{i}=\beta_{i}\left(-\sum_{i=1}^{N} \Delta P_{i}\right) \\
& \sum_{i=1}^{N} \beta_{i}=1 \\
& \sum_{i=1}^{N} \Delta P_{i}=\sum_{i=1}^{N} \Delta G_{i} \\
& \\
& \\
& \\
&
\end{array}
$$

where $N$ is the number of generators in the system; $G_{i}$ is the generation power output; $D_{i}$ is the load amount; $P_{i}$ is the forecasted wind power; $c_{i}$ is the offer price of generation; $G_{\text {exp }, i}$ is the expected generation power output; $P_{\text {exp }, i}$ is the expected wind power; $\Delta G_{i}$ is the generation response to the wind uncertainty; $\Delta P_{i}$ is the uncertain output of wind power; $\beta_{i}$ is the sharing factor of generation for the wind uncertain power output; $G S F_{l, i}$ is the system generation shift factor; $L U_{l}$ is the transmission limit; $G_{i, \max }$ and $G_{i, \min }$ are the generation upper and lower limits, respectively; (3)-(6) are the chance constraints considering the impact of the wind uncertainty on the transmission overload and generation output violation; $\epsilon$ is the confidence level in the chance constraints (mostly between $1 \%$ and 5\%). The decision variable in the model is the generation dispatch $G_{\text {exp }, i}$ and the balancing factor $\beta_{i}$.

Define that the uncertain part of wind power output $\Delta \boldsymbol{P}$ has the mean as $\boldsymbol{\mu}$ and covariance as $\boldsymbol{\Sigma}$ which is a positive semidefinite matrix. In addition, define $\boldsymbol{a}_{l}(\boldsymbol{\beta})$ as a onecolumn matrix whose element $a_{l, i}(\boldsymbol{\beta})$ is given by (12), and $\boldsymbol{b}_{i}(\boldsymbol{\beta})$ as a one-column matrix given by (13).

$a_{l, i}(\boldsymbol{\beta})=-\sum_{k=1}^{N} G S F_{l, k} \cdot \beta_{k}+G S F_{l, i}$

$\boldsymbol{b}_{i}(\boldsymbol{\beta})=\underbrace{\left[\begin{array}{lll}\beta_{i} & \cdots & \beta_{i}\end{array}\right]^{\mathrm{T}}}_{W}$

where $W$ is the number of wind power plants.

Then, introduce auxiliary variables $\eta_{P F, l}$ and $\eta_{g, i}$ as shown in (14) and (15).

$\sqrt{\boldsymbol{a}_{l}(\boldsymbol{\beta})^{\mathrm{T}} \boldsymbol{\Sigma} \boldsymbol{a}_{l}(\boldsymbol{\beta})} \leq \eta_{P F, l}$

$\sqrt{\boldsymbol{b}_{i}(\boldsymbol{\beta})^{\mathrm{T}} \boldsymbol{\Sigma} \boldsymbol{b}_{i}(\boldsymbol{\beta})} \leq \eta_{g, i}$

Equations (14) and (15) can be formulated as secondorder cone constraints as:

$$
\begin{aligned}
& \left\|\Sigma^{\frac{1}{2}} \boldsymbol{a}_{l}(\boldsymbol{\beta})\right\|_{2} \leq \eta_{P F, l} \\
& \left\|\Sigma^{\frac{1}{2}} \boldsymbol{b}_{i}(\boldsymbol{\beta})\right\|_{2} \leq \eta_{g, i}
\end{aligned}
$$

The CC-OPF model is then formulated as (1), (12), (13), (16)-(22).

$\sum_{i=1}^{N}\left(G_{\exp , i}+P_{\exp , i}\right)-\sum_{i=1}^{N} D_{i}=0$

$-\sum_{i=1}^{N}\left[G S F_{l, i} \cdot\left(G_{e x p, i}+P_{e x p, i}-D_{i}\right)+a_{l, i}(\boldsymbol{\beta}) \mu_{i}\right]$

$-K_{\epsilon} \eta_{P F, l} \geq-L U_{l}$

$\sum_{i=1}^{N}\left[G S F_{l, i} \cdot\left(G_{\text {exp }, i}+P_{\text {exp }, i}-D_{i}\right)+a_{l, i}(\boldsymbol{\beta}) \mu_{i}\right]-K_{\epsilon} \eta_{P F, l} \geq$ $-L U_{l}$

$-G_{i}-\beta_{i} \sum_{k=1}^{N} \mu_{k}-K_{\epsilon} \eta_{g, i} \geq-G_{i, \max }$

$G_{i}+\beta_{i} \sum_{k=1}^{N} \mu_{k}-K_{\epsilon} \eta_{g, i} \geq G_{i, \min }$

where $K_{\epsilon}$ is the coefficient to control the robustness of the chance constraints to wind power forecasting errors. If the forecasting error follows a Gaussian distribution [5], the value of $K_{\epsilon}$ is decided by (23).

$K_{\epsilon}=\Psi^{-1}(1-\epsilon)$

where $\Psi(x)$ is the cumulative distribution function (CDF) of Gaussian distribution. 


\section{ADRCC-OPF}

In (23), $K_{\epsilon}$ is obtained based on the Gaussian distribution assumption for the wind power forecasting errors, which is not always the case in actual practice, as shown in Fig. 1 [4]. This section discusses the proposed ADRCC-OPF model that does not need the Gaussian distribution assumption for wind power forecasting errors and can control the tradeoff between the reliability and the system cost.

First, the assumption is that the first- and second-order moments of the uncertain variables (mean and covariance) are estimated based on the historical data [2]. A set $\boldsymbol{p}$ of all the possible distributions $\boldsymbol{P}$ satisfying the mean and covariance values are represented as:

$\boldsymbol{p}=\left\{\boldsymbol{P} \in P_{0}\left(\mathbf{R}^{|v|}\right): \boldsymbol{E}_{\boldsymbol{P}}[\boldsymbol{\omega}]=\boldsymbol{\mu}, \boldsymbol{E}_{\boldsymbol{P}}\left[\omega \boldsymbol{\omega}^{\mathrm{T}}\right]=\boldsymbol{\Sigma}\right\}$

where $\omega$ is the uncertain variable (here is the wind power forecasting error); $P_{0}\left(\mathbf{R}^{|v|}\right)$ denotes the set of all of the probabilistic distributions on $\mathbf{R}^{|v|}$ with mean as $\boldsymbol{\mu}$ and covariance matrix as $\Sigma ; \boldsymbol{E}_{\boldsymbol{P}}[\boldsymbol{\omega}]$ represents the expectation of $\omega[3,6,7]$.

When only the mean and covariance of the distribution are obtained from the historical data, for $\epsilon \in(0,1)$, the distributionally robust chance constraint is formulated with $K_{\epsilon}$ value decided by (25) [1].

$K_{\epsilon}=\sqrt{(1-\epsilon) / \epsilon}$

If the forecasting error distribution is symmetrical with observing the historical forecasting error data, then the DRCC-OPF is formulated [1] with $K_{\epsilon}$ value decided by (26).

$K_{\epsilon}=\sqrt{1 /(2 \epsilon)}$

The values of $K_{\epsilon}$ in the three cases are listed in Table 1 for $\epsilon$ varying from $2 \%$ to $5 \%$.

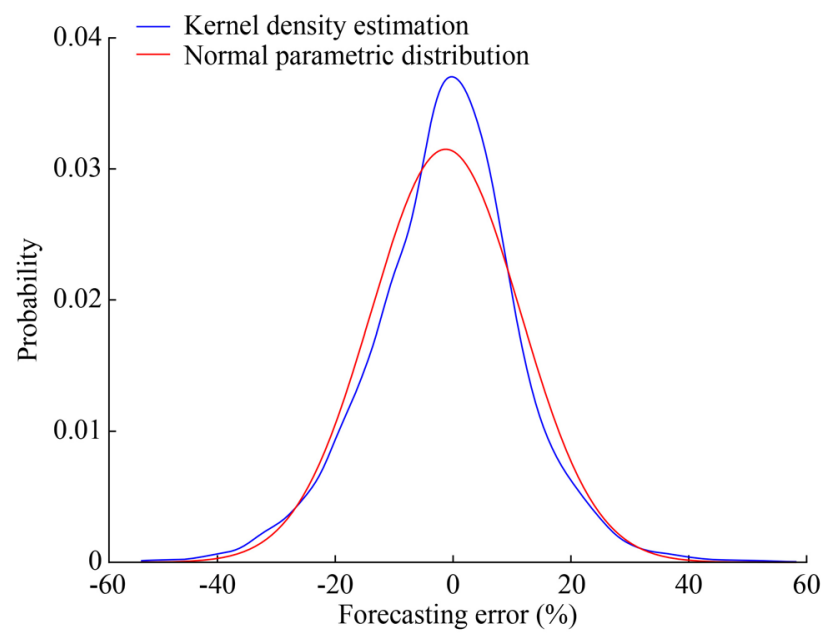

Fig. 1 Wind power forecasting error distribution
However, the actual wind power forecasting errors might not be Gaussian or symmetrical. Therefore, using the $K_{\epsilon}$ listed in Table 1 might lead to over-optimistic or overconservative results. Using the $K_{\epsilon}$ values of the distributionally robust case will lead to over-conservative results that has a higher operating cost and is not likely to be adopted by the system operators. Therefore, it is important to choose an appropriate $K_{\epsilon}$ value leading to a predefined reliability and system cost tradeoff.

For the given $\epsilon$ value under each distribution assumption (Gaussian, symmetric distributionally robust and distributionally robust), the $\mathrm{CC}-\mathrm{OPF}$ is performed to obtain the dispatch results and the balancing factors using $K_{\epsilon}$ values listed in Table 1. With the actual historical wind power forecasting error data, the system actual violations under each distribution assumption are obtained. Comparing the obtained violation value with the predefined value $(\epsilon)$, the operators can choose the adjustable coefficient as shown in (27) to make the system violation value as close to the predefined value as possible to avoid over-optimistic or over-conservative results shown in Fig. 2.

$K_{\epsilon}=K_{\epsilon 1}+\left(\epsilon-V_{1}\right) \frac{K_{\epsilon 2}-K_{\epsilon 1}}{V_{2}-V_{1}}$

where $K_{\epsilon 1}$ and $K_{\epsilon 2}$ are the coefficients leading to the violation higher and lower than the predefined value, respectively; $V_{1}$ and $V_{2}$ are the realized violation values. Here a linear approximation is used for simplicity because the forecasting errors have a linear relationship with $K_{\epsilon}$ shown in the chance constraints in (19)-(21).

Note that after obtaining the generation dispatch and the balancing factors, the historical forecasting error data are used to calculate the system violation. In this process, the computation procedure can be paralleled to obtain the violation values under different distribution assumption fast and efficiently.

In the system operation, the system operators can choose the appropriate $K_{\epsilon}$ based on the actual historical data for each operation interval using the actual system realized wind power deviation. Then in the actual operation, they can choose the appropriate coefficients based on the offline simulation. Similar mechanism can be found in Electricity Reliability Council of Texas (ERCOT) dynamic reserve curve [8].

\section{Case studies}

The test system has been modified from the PJM 5-bus system depicted in Fig. 3. The numbers besides the generators represent the generation offer price (\$/MWh) and capacity (MW). The system parameters are from [9]. In this study, the peak load in this system is $1500 \mathrm{MW}$ in the 
Table $1 K_{\epsilon}$ values under different distribution assumptions

\begin{tabular}{|c|c|c|c|}
\hline \multirow[t]{2}{*}{$\epsilon(\%)$} & \multicolumn{3}{|l|}{$K_{\epsilon}$} \\
\hline & Gaussian & $\begin{array}{l}\text { Symmetric } \\
\text { distributionally robust }\end{array}$ & $\begin{array}{l}\text { Distributionally } \\
\text { robust }\end{array}$ \\
\hline 2 & 2.0538 & 5.0000 & 7.0000 \\
\hline 3 & 1.8810 & 4.0825 & 5.6862 \\
\hline 4 & 1.7507 & 3.5355 & 4.8990 \\
\hline 5 & 1.6450 & 3.1623 & 4.3589 \\
\hline
\end{tabular}

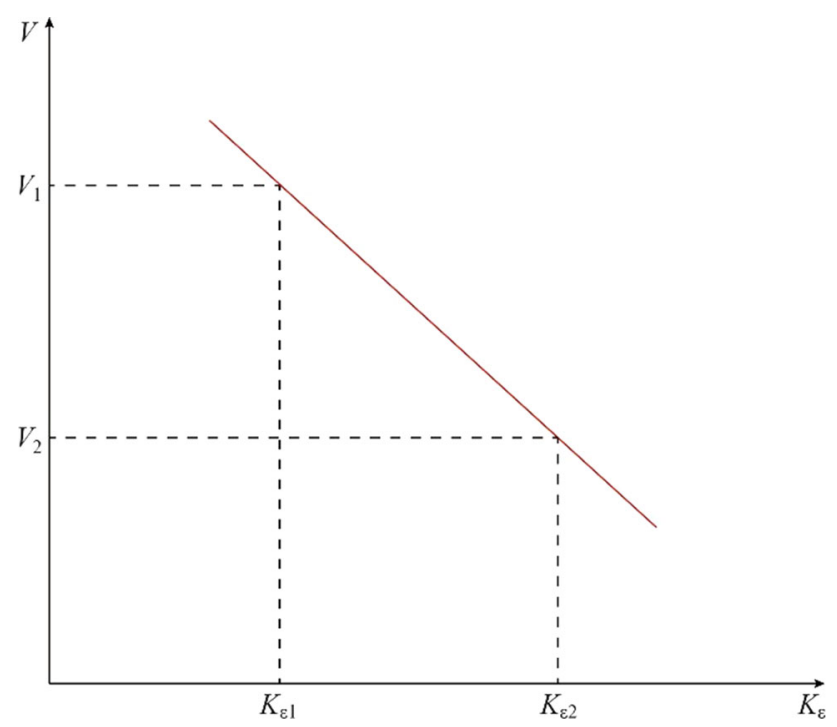

Fig. 2 Illustration of $K_{\epsilon}$ calculation

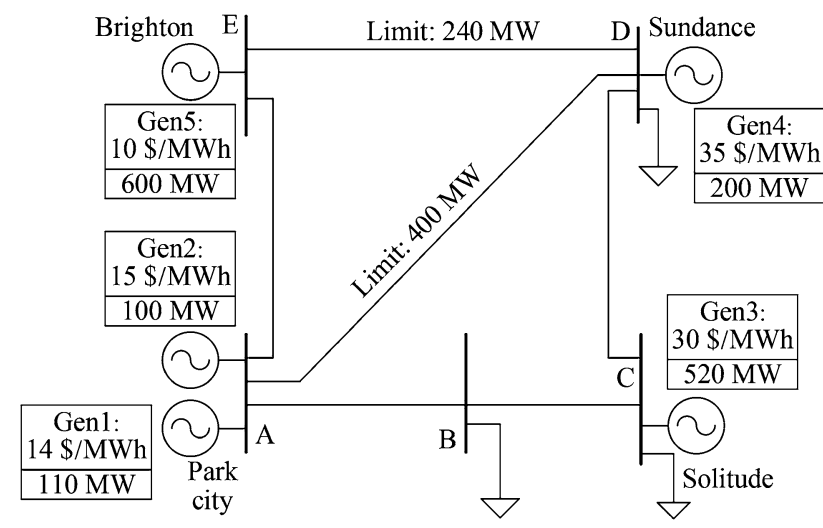

Fig. 3 PJM 5-bus system and generation parameters

studied interval, and the total load is equally distributed among buses B, C, and D. Two wind power plants with 300 MW as their forecasted mean power output are located at buses B and C. One-year 8760-hour historical wind forecasting error data are utilized from California Independent System Operator (CAISO) to obtain the forecasting error mean and covariance [4], which are also utilized to examine the system violation with DRCC-OPF.
Table 2 System generation cost under different distribution assumptions

\begin{tabular}{llll}
\hline$\epsilon(\%)$ & \multicolumn{2}{l}{ System generation cost $(\$)$} \\
\cline { 2 - 4 } & Gaussian & $\begin{array}{l}\text { Symmetric } \\
\text { distributionally robust }\end{array}$ & $\begin{array}{l}\text { Distributionally } \\
\text { robust }\end{array}$ \\
\hline 2 & 15170.8 & 15716.3 & 16332.5 \\
3 & 15138.7 & 15546.6 & 15882.8 \\
4 & 15114.5 & 15445.4 & 15697.7 \\
5 & 15094.8 & 15376.3 & 15597.7 \\
\hline
\end{tabular}

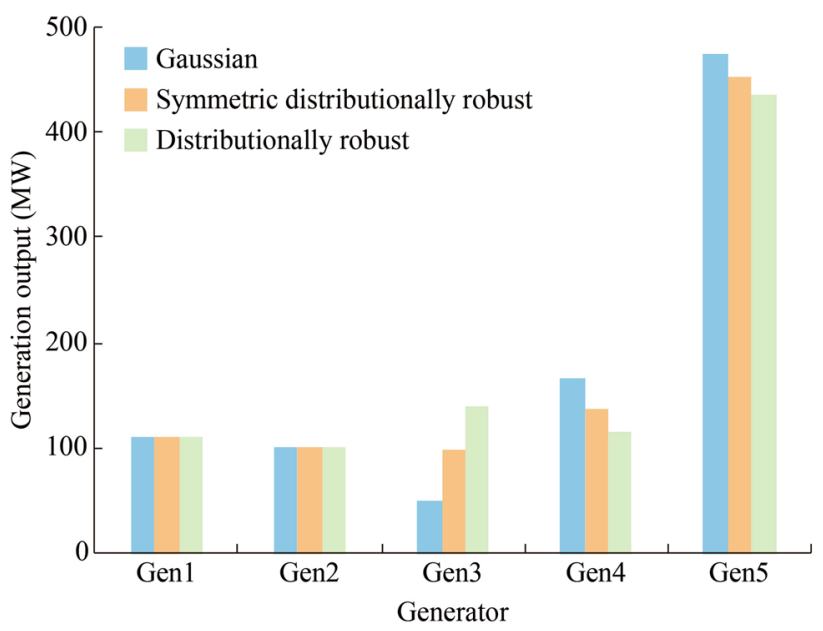

Fig. 4 Generation dispatch under different distribution assumptions

Table 2 lists the system generation cost with different distribution assumptions and confidential levels from $2 \%$ to $5 \%$. It is obvious that the cost increases with the confidence level. In Gaussian distribution assumption, the cost increase from $\$ 15094.8$ to $\$ 15170.8$ when $\epsilon$ changes from $5 \%$ to $2 \%$. In the distributionally robust case, the cost increases from $\$ 15597.7$ to $\$ 16332.5$. In addition, the cost increases with the robustness of the wind power forecasting error distribution assumption. For instance, the cost in the distributionally robust case is the highest, the symmetrically distributionally robust case has the second highest cost, and the Gaussian distribution case has the lowest generation cost. It is clear from Table 1 that higher confidence level (lower $\epsilon$ ) and higher robustness of wind power forecasting error distribution lead to a higher $K_{\epsilon}$. However, this comes at a cost.

Figure 4 shows the generation dispatch with $\epsilon$ of 5\%. The generation changes with the conservativeness of the wind power forecasting error. In the distributionally robust case, Gen 4 and Gen5 reduce their power outputs while Gen3 increases its power to mitigate the wind power uncertainty. This leads to the increment of system 
Table 3 Violation probability of Gen4 under different distribution assumptions

\begin{tabular}{lllll}
\hline$\epsilon(\%)$ & \multicolumn{2}{l}{ Violation probability of Gen4 (\%) } & \multirow{2}{*}{$K_{\epsilon}$} \\
\cline { 2 - 4 } & Gaussian & $\begin{array}{l}\text { Symmetric } \\
\text { distributionally robust }\end{array}$ & $\begin{array}{l}\text { Distributionally } \\
\text { robust }\end{array}$ & \\
\hline 2 & 3.27 & 0.021 & 0 & 3.205 \\
3 & 4.40 & 0.169 & 0 & 2.609 \\
4 & 5.43 & 0.350 & 0.021 & 2.253 \\
5 & 6.25 & 0.630 & 0.106 & 1.982 \\
\hline
\end{tabular}

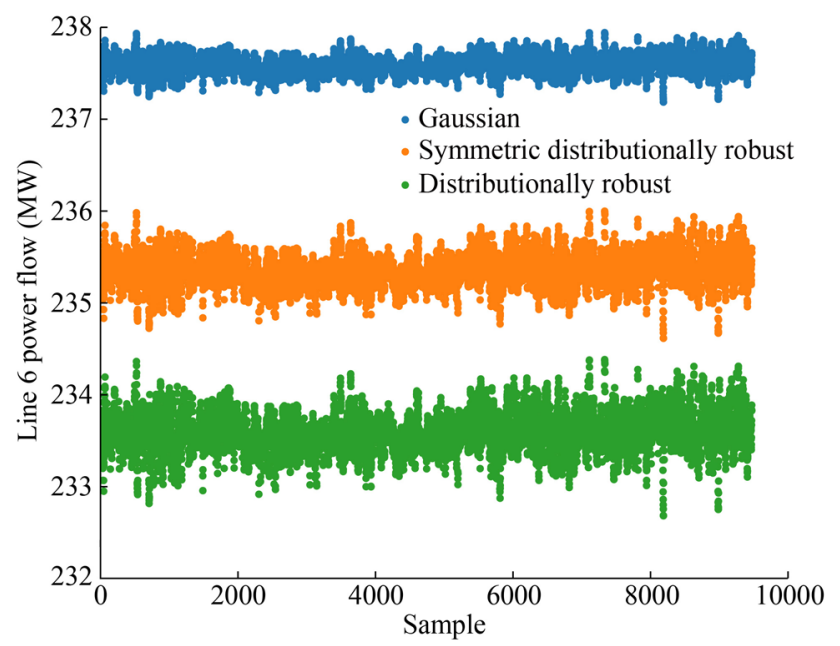

Fig. 5 Power flow of line 6 considering wind forecasting errors under different distributions

generation cost, since Gen3 has a higher cost than Gen5, as shown in Fig. 3.

After the generation dispatch and sharing factors are obtained from the proposed ADRCC-OPF, the historical 8760 wind power forecasting error scenarios are utilized to represent the actual wind power realization and the system violation such as generation violation is calculated [10]. Table 3 shows the output violation of Gen 4 under different confidence levels and various distribution assumptions. Figures 5 and 6 are the power flow realization of line 6 and the power output of Gen 4 considering the wind power forecasting error.

Table 3 demonstrates that the Gaussian distribution assumption will lead to an optimistic result. For instance, when the confidence level is $1 \%$, Gen 4 has a $4.64 \%$ probability of violating its generation upper limit. In actual system operation, this will bring risk to system operations. The symmetrically distributionally robust and distributionally robust cases lead to a conservative dispatch because the actual violation possibility of Gen 4 is lower

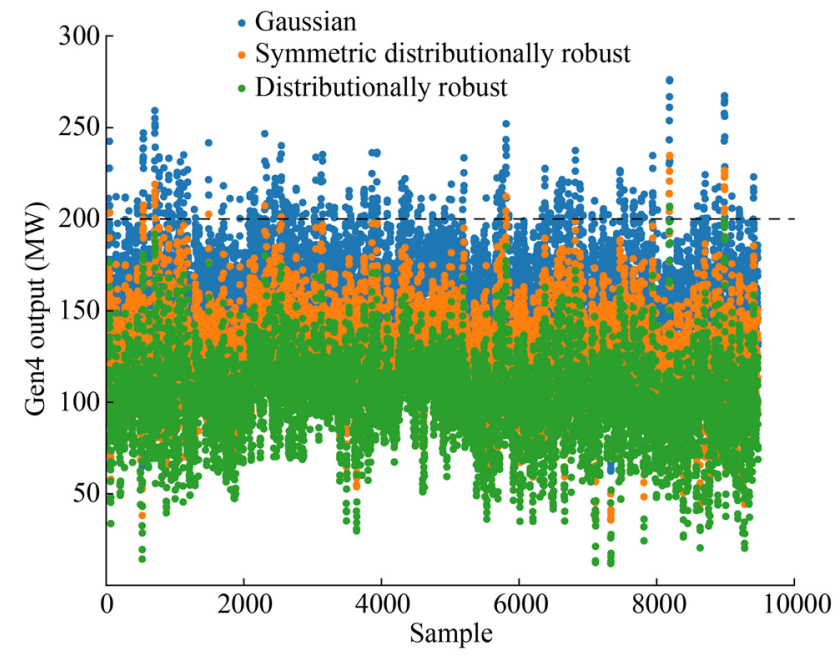

Fig. 6 Output of Gen4 considering wind forecasting errors under different distributions

than the predefined $\epsilon$ value in these cases. This over-conservativeness leads to a higher generation cost. Therefore, appropriately choosing the distribution assumption of wind power forecasting error (the value of $K_{\epsilon}$ ) to represent the predefined risk level is of importance for the system operators to tradeoff between the system reliability and the cost. Again, this is also the reason that the proposed method is adjustable in addition to being distributionally robust. In this table, the adjustable coefficients $K_{\epsilon}$ leading to the violation probability close to the predefined $\epsilon$ value are also listed. The value of $K_{\epsilon}$ is obtained using (27). The violation in Gaussian distribution is $V_{1}$, and the violation in symmetrical distributionally robust case is $V_{2}$. The values of $K_{\epsilon}$ in Table 1 for Gaussian and symmetrical distributionally robust are $K_{\epsilon 1}$ and $K_{\epsilon 2}$.

In Fig. 5, it is obvious that the transmission power flow will reduce to a narrow range, and that the maximum potential power flow realization reduces, which also leads to lower transmission power flow violations. The black dashed line in Fig. 6 is the upper limit of Gen4. Figure 6 clearly shows that with the conservativeness increases the generation power output will reduce which leads to a lower generation output violation.

The IEEE 118-bus system is tested to verify the proposed model in a large-scale system. The system data is from $[9,11]$. Four wind power plants are connected at bus 2 , bus 5 , bus 53 , and bus 86 with the expected power output equal to $300 \mathrm{MW}$ for the studied interval. The confidence level is $5 \%$. The system generation cost and output violation probabilities of Gen 2 are listed in Table 4. In this case, the $K_{\epsilon}$ that leads to the closest value to the defined confidence level should be within the values of the symmetrically distributionally robust case and the distributionally robust case in Table 1. 
Table 4 System results under different distribution assumptions

\begin{tabular}{lll}
\hline Distribution assumption & $\begin{array}{l}\text { Total } \\
\text { generation } \\
\text { cost }(\$)\end{array}$ & $\begin{array}{l}\text { Violation } \\
\text { probability } \\
\text { of Gen2 }(\%)\end{array}$ \\
\hline Gaussian & 50702 & 19.25 \\
Symmetric distributionally robust & 53687 & 8.83 \\
Distributionally robust & 56842 & 0 \\
\hline
\end{tabular}

\section{Conclusion}

This work proposes a new chance-constrained OPF model called ADRCC-OPF. The influence of the distributional robustness for wind power forecasting on the generation cost and system violations is studied. It shows that the Gaussian distribution assumption for wind power forecasting can lead to overoptimistic results for the system violations and bring risk to system operation. The traditional distributionally robust CC-OPF model will lead to over-conservative results with a high operating cost. It is demonstrated in this work that the proposed ADRCC-OPF can achieve an appropriate tradeoff between the conservativeness and the system cost when an appropriate adjustable coefficient is chosen to control the robustness of the chance constraints.

Acknowledgements This work was co-authored by Alliance for Sustainable Energy, LLC, the manager and operator of the National Renewable Energy Laboratory for the U.S. Department of Energy (DOE) (No. DE-AC36-08GO28308). The funding was provided by U.S. DOE Office of Energy Efficiency and Renewable Energy Wind Energy Technologies Office. The views expressed in the article do not necessarily represent the views of the DOE or the U.S. Government. The U.S. Government retains and the publisher, by accepting the article for publication, acknowledges that the U.S. Government retains a nonexclusive, paid-up, irrevocable, worldwide license to publish or reproduce the published form of this work, or allow others to do so, for U.S. Government purposes.

Open Access This article is distributed under the terms of the Creative Commons Attribution 4.0 International License (http:// creativecommons.org/licenses/by/4.0/), which permits unrestricted use, distribution, and reproduction in any medium, provided you give appropriate credit to the original author(s) and the source, provide a link to the Creative Commons license, and indicate if changes were made.

\section{References}

[1] Calafiore GC, El Ghaoui L (2006) On distributionally robust chance-constrained linear programs. J Optim Theory Appl 130(1):1-22

[2] Jiang R, Guan Y (2016) Data-driven chance constrained stochastic program. Math Programm 158(1-2):291-327
[3] Xie W, Ahmed S (2018) Distributionally robust chance constrained optimal power flow with renewables: a conic reformulation. IEEE Trans Power Syst 33(2):1860-1867

[4] Hodge BM, Florita A, Orwig K et al (2012) A comparison of wind power and load forecasting error distributions. In: Proceedings of 2012 world renewable energy forum, Denver, USA, 13-17 May 2012, pp 1-8

[5] Jabr RA (2013) Adjustable robust OPF with renewable energy sources. IEEE Trans Power Syst 28(4):4742-4751

[6] Fang X, Hodge BM, Du E et al (2018) Modelling wind power spatial-temporal correlation in multi-interval optimal power flow: a sparse correlation matrix approach. Appl Energy 230:531-539

[7] Fang X, Hodge BM, Du E et al (2019) Introducing uncertainty components in locational marginal prices for pricing wind power and load uncertainties. IEEE Trans Power Syst. https:// doi.org/10.1109/tpwrs.2018.2881131

[8] ERCOT market training website. http://www.ercot.com/content/ wcm/training_courses/107/ordc_workshop.pdf. Accessed 1 May 2018

[9] Fang X, Li F, Wei Y et al (2016) Strategic scheduling of energy storage for load serving entities in locational marginal pricing market. IET Gen Transm Distrib 10(5):1258-1267

[10] Hodge BM, Milligan M (2011) Wind power forecasting error distributions over multiple timescales. In: Proceedings of IEEE PES general meeting, Detroit, USA, 24-29 July 2011, pp 1-8

[11] Fang X, Hu Q, Li F et al (2016) Coupon-based demand response considering wind power uncertainty: a strategic bidding model for load serving entities. IEEE Trans Power Syst 31(2):1025-1037

Xin FANG received his B.S. degree from Huazhong University of Science and Technology, China in 2009, M.S. degree from China Electric Power Research Institute in 2012, and Ph.D. degree from the University of Tennessee, Knoxville (UTK), USA in 2016. He is currently with the National Renewable Energy Laboratory (NREL). His research interests include electricity market, power system planning and optimization, renewable energy integration, and demand response.

Bri-Mathias HODGE received the B.S. degree in chemical engineering from Carnegie Mellon University, USA in 2004, the M.S. degree from the Process Design and Systems Engineering Laboratory of Abo Akademi, Turku, Finland, in 2005, and the Ph.D. degree in chemical engineering from Purdue University, USA in 2010. He is currently the Manager of the Power System Design and Studies Group at the National Renewable Energy Laboratory, Golden, USA. His current research interests include energy systems modeling, simulation, optimization, and wind power forecasting.

Fangxing LI is also known as Fran LI. He received the B.S.E.E. and M.S.E.E. degrees from Southeast University, China in 1994 and 1997, respectively, and the Ph.D. degree from Virginia Tech, USA in 2001. $\mathrm{He}$ is currently the James McConnell Professor in electrical engineering and the Campus Director of the CURENT research center at the University of Tennessee, Knoxville, USA. Prior to joining UTK, he worked at ABB Consulting as a Senior Engineer and then as a Principal Engineer from 2001 to 2005. His current research interests include renewable energy integration, demand response, power markets, distributed generation, measurement-based technology and power system computing.

Ershun DU received both a B.S. and Ph.D. from the Electrical Engineering Department of Tsinghua University, China in 2013 and 
2018, respectively. His research interests include renewable energy uncertainty analysis, power system economics and planning with renewable energy.

Chongqing KANG received his Ph.D. from the Electrical Engineering Department of Tsinghua University, China in 1997. He is now a
Professor at the same university. His research interests include lowcarbon electricity, power system planning, renewable energy, power markets and power system load forecasting. 